\title{
$z>4$ low luminosity dusty galaxy candidates in the Frontier Fields A2744, AS1063 and A370
}

\author{
Frédéric Boone $^{1}$, Daniel Schaerer ${ }^{2}$, Johan Richard ${ }^{3}$, Benjamin \\ Clément $^{3}$, Eiichi Egami ${ }^{4}$, Tim Rawle ${ }^{5}$, Dieter Lutz ${ }^{6}$, Axel Weiss ${ }^{7}$, \\ Johannes G. Staguhn ${ }^{8}$ and HLS team \\ ${ }^{1}$ Université Paul Sabatier, CNRS, IRAP, 14 Avenue E. Belin, 31400, Toulouse, France \\ email: frederic.boone@irap.omp.eu \\ ${ }^{2}$ Observatoire de Genève, Département d'Astronomie, Université de Genève, 51 Ch. des \\ Maillettes, 1290, Versoix, Switzerland \\ ${ }^{3}$ CRAL, Observatoire de Lyon, Université de Lyon 1, 9 avenue Ch. André, F-69561 \\ Saint-Genis Laval, France \\ ${ }^{4}$ Steward Observatory, University of Arizona, 933 N. Cherry Ave, Tucson, AZ 85721, USA \\ ${ }^{5}$ ESA / Space Telescope Science Institute (STScI), 3700 San Martin Drive, Baltimore, MD \\ 21218, USA \\ ${ }^{6}$ Max-Planck-Institut für extraterrestrische Physik, 85741 Garching, Germany \\ ${ }^{7}$ Max-Planck-Institut für Radioastronomie, Auf dem Hügel 69, 53121, Bonn, Germany \\ ${ }^{8}$ Observational Cosmology Lab, Code 665, NASA Goddard Space Flight Center, Greenbelt, \\ MD 20771, USA
}

\begin{abstract}
We have conducted a LABOCA $870 \mu \mathrm{m}$ follow-up of ten massive lensing clusters of the Herschel Lensing Survey (HLS, Egami et al. 2010) aiming at unveiling the yet hidden part of dusty star formation in the distant Universe. Among these clusters, A2744 and AS1063 are part of the Frontier Fields HST program. We also obtained $2 \mathrm{~mm}$ bolometer observations of A2744 and A370 with the GISMO array at the IRAM $30 \mathrm{~m}$. We detected sources that are undetected with Herschel (PACS and SPIRE) implying either a very high redshift $(z>4)$ or a very low dust temperature $(T<25 \mathrm{~K})$. Their flux also imply a low intrinsic luminosity, $L_{\mathrm{FIR}}<10^{12} L_{\odot}$. Some of them are extended and could correspond to multiple sources or to multiple images of a lensed source. Substructures in the Sunyaev-Zeldovich effect could also contribute this very red submm emission. An ALMA program is ongoing to unveil the nature of these sources.
\end{abstract}

Keywords. Keyword1, keyword2, keyword3, etc.

\section{Observations and Results}

We have undertaken a $870 \mu \mathrm{m}$ follow-up of ten clusters (MS2137, A2667, RXCJ0237.4, RXCJ0118.1, RXCJ0307.0, A2744, AC114, RXCJ0304.1, AS1063 and RXCJ0638.7) of the Herschel Lensing Survey (HLS, Egami et al. 2010) with the APEX/LABOCA bolometer array in a large program (PI: Boone and Lutz). In addition we observed two FF clusters, A2744 and A370 with the GISMO/IRAM $30 \mathrm{~m}$ bolometer array at $2 \mathrm{~mm}$.

In the fields of A2744 (Fig. 1), AS1063, MS2137, A2667 and A370, we detect sources that are undetected or at low significance in Herschel (PACS and SPIRE) maps implying either $z>4$ or $T<25 \mathrm{~K}$. They are expected to have lower intrinsic luminosities than the flux-selected SMGs (H-ATLAS and SPT samples) or than blank field SMGs (e.g., LESS sample). Some of them are extended and could correspond to multiple sources or to multiple images of a lensed source. 

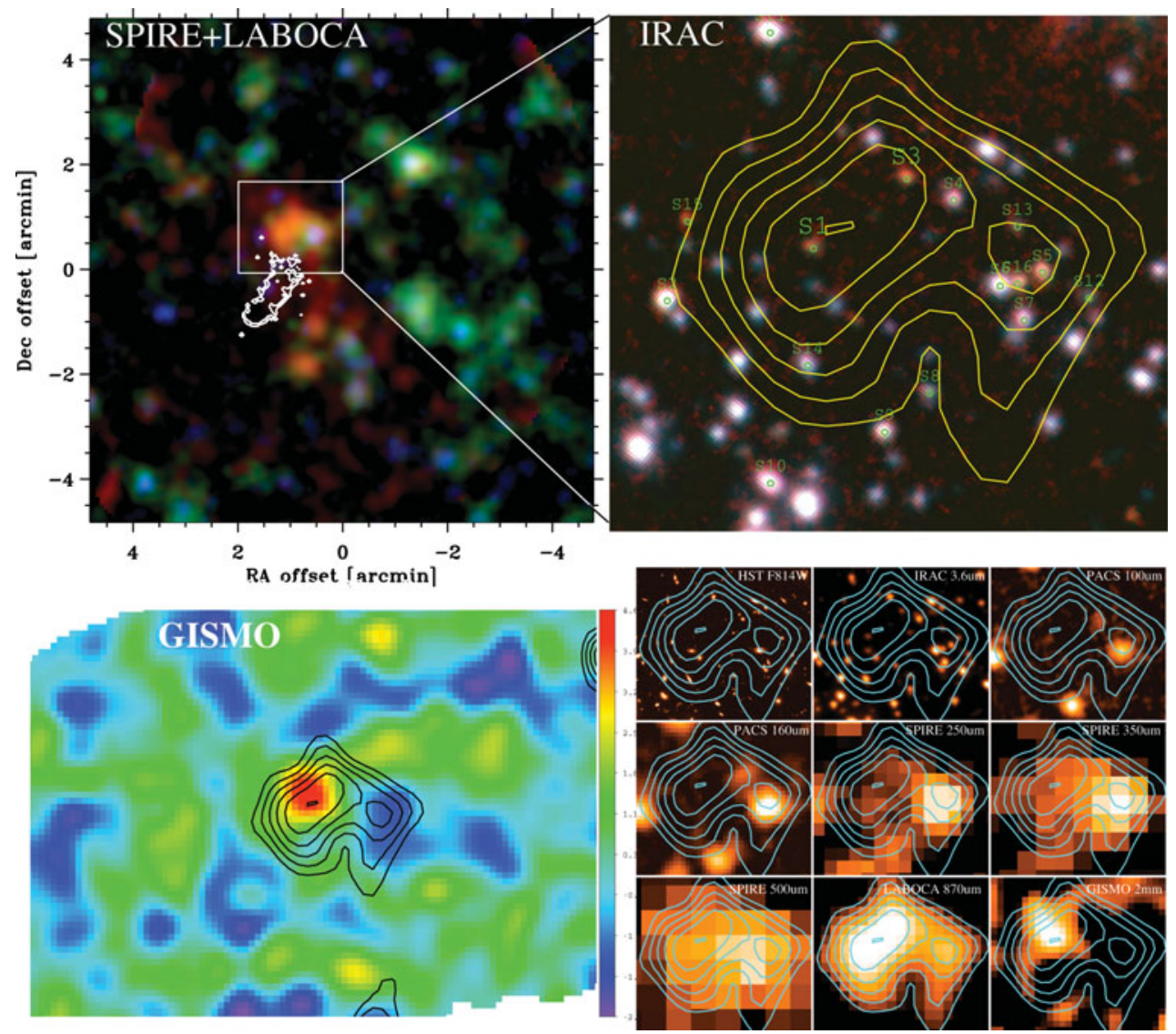

Figure 1. Upper Left: SPIRE 250 (blue), 500 (green), and LABOCA $870 \mu \mathrm{m}$ (red) composite image of Abell 2744. The contours in white correspond to the cluster critical line leading to a maximal magnification factor of 1.8 for the sources detected in the extended submm LABOCA emission. Upper Right: Spitzer/IRAC 3.6 (blue), 4.5 (green) and $5.8 \mu \mathrm{m}$ (red) composite image of the region where the $870 \mu \mathrm{m}$ emission is the strongest (in yellow contours with levels starting at $3 \sigma$ and spaced by $2 \sigma$ with $\sigma=1.2 \mathrm{mJy}$ ). We identify several sources (labeled $\mathrm{S} 1$ to $\mathrm{S} 15$ ) detected by both IRAC and MIPS $(24 \mu \mathrm{m})$ that could contribute to the submm emission. S1 and S3 would be the main contributors to the 'red' submm emission. Lower Left: The $2 \mathrm{~mm}$ continuum map obtained with IRAM-30m/GISMO overlaid with the same LABOCA contours in black. The GISMO peak flux is $1.2 \mathrm{mJy}$. Lower Right: Thumbnails showing the region of interest at different wavelengths. From left to right and from top to bottom the wavelengths are: $0.814,3.6,100,160,250,350,500,870,2000 \mu \mathrm{m}$.

In particular, we found a very red submm source in AS1063 that may be associated with a multiple-image system at $z=6.107$ (Boone et al. 2013).

These sources may bridge the gap between galaxies traditionnally selected in the submm and optically selected galaxies. Alternatively they may correspond to substructures in the Sunyaev-Zeldovich effect due to shocks in the clusters.

ALMA and NOEMA follow up are ongoing to unveil the nature of these exceptionally red submm sources.

\section{References}

Boone, F., Clément, B., Richard, J., et al. 2013, A\&A, 559, L1

Egami, E., Rex, M., Rawle, T. D., et al. 2010, A\&SA, 518, L12+ 